\title{
Semi-automated analysis of infarct heterogeneity on DE-MRI using graph cuts
}

\author{
YingLi Lu ${ }^{1}$, Kim A Connelly ${ }^{2,3^{*}}$, Yuesong Yang ${ }^{1}$, Subodh B Joshi ${ }^{2,3}$, Graham Wright ${ }^{1,4}$, Perry E Radau ${ }^{1}$ \\ From 15th Annual SCMR Scientific Sessions \\ Orlando, FL, USA. 2-5 February 2012
}

\begin{abstract}
Background
Two popular methods for determining the threshold values for the infarct core and gray zone on delayed enhancement MR images (DE-MRI) have been proposed previously: full width and half maximum (FWHM) and standard deviation (SD) methods [1]. Major limitations of these methods are:1) three manually drawn contours are needed for endocardial, epicardial and remote myocardium boundaries, which is time consuming and suffers from inter-observer and intra-observer variability; 2) the difficulty in reproducible manual delineation of remote myocardium, is an important contributor to variability in results; and 3) the dependence on the remote region statistics is problematic due to the low SNR of this region [2]. The purpose of this research was to develop a novel algorithm for segmentation of infarct core and gray zone from conventional IR-GRE shortaxis MR images with highly robust and reproducible results comparable to the FWHM analysis while eliminating the requirement for drawing a remote myocardial region.
\end{abstract}

\section{Methods}

Eleven male patients (age: $63.5 \pm 11.8 \mathrm{yr}$ ) with known CAD and evidence of LGE and chronic MI had cardiac IR-GRE MR scans with full left ventricle (LV) coverage (7-13 slices). MR imaging was performed on a $1.5 \mathrm{~T}$ scanner (CV/i, GE Healthcare) using an 8-channel cardiac coil. DE-MRI was started $10 \mathrm{~min}$ after the injection of $0.2 \mathrm{mmol} / \mathrm{kg}$ of Gd-DTPA (Magnevist, Berlex). First, the endocardial and epicardial contours were generated from the corresponding SSFP images automatically [3] with papillary muscles included. $(42 \%(54 / 136)$ of the contours need manual adjustment. Next, the graph cuts

${ }^{2}$ Keenan Research Centre in the Li Ka Shing Knowledge Institute, St. Michael's Hospital and University of Toronto, Toronto, ON, Canada Full list of author information is available at the end of the article algorithm [4] was used to segment the infarct: 1) a twoclasses Gaussian Mixture Model (GMM) was created for the myocardial ROI, determined by the endocardial and epicardial contours; 2) each pixel was assigned to the most likely Gaussian component; and 3) a graph was built and the graph cut algorithm finds the optimum classification of healthy and infarcted myocardium. Finally, the segmented infarct is separated into infarct core and gray zone using a threshold of half the maximal signal within the segmented infarct (Fig. 1). Linear regression analysis and Bland-Altman plots were used to compare the FWHM method (requiring manual ROIs for LV and remote myocardium) and our method (Fig. 2).

\section{Results}

There were excellent correlations of the infarct size (infarct core $1: \mathrm{R}^{\wedge} 2=0.99$; gray zone: $\mathrm{R}^{\wedge} 2=0.95$ ) derived from our graph cuts method and the manual FWHM method. The Bland-Altman analysis indicated that there was a small overestimation bias (infarct core: $0.17 \mathrm{~g}$; gray zone: $0.68 \mathrm{~g}$ ) with limits of agreement of \pm $1.40 \mathrm{~g}$ (infarct core) and $\pm 4.27 \mathrm{~g}$ (gray zone). This variability is small relative to the reported range of gray zone masses $(20+/-13 \mathrm{~g}, \mathrm{~N}=91$ [2]).

\section{Conclusions}

The results for the proposed semi-automated segmentation technique indicate that it will streamline accurate quantification of myocardial infarct on IR-GRE MR infarct images in clinical practice.

\section{Funding}

The authors thank the Canadian Foundation for Innovation (CFI), the Canadian Institutes of Health Research (CIHR), and MaRS Innovation Proof of Principle Program for their support. 


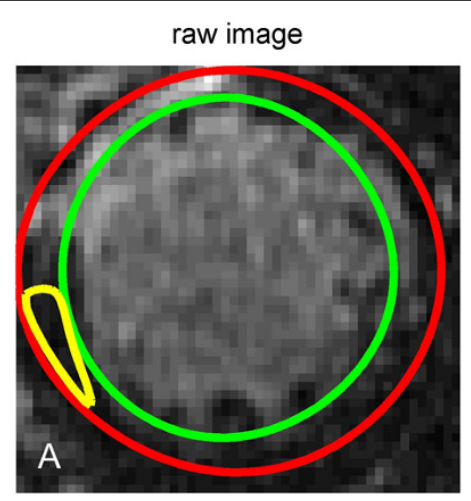

Graph cuts

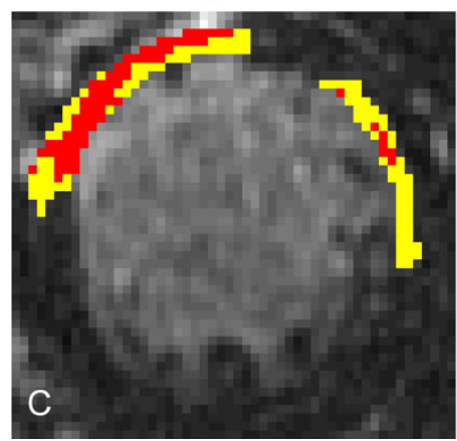

roi image

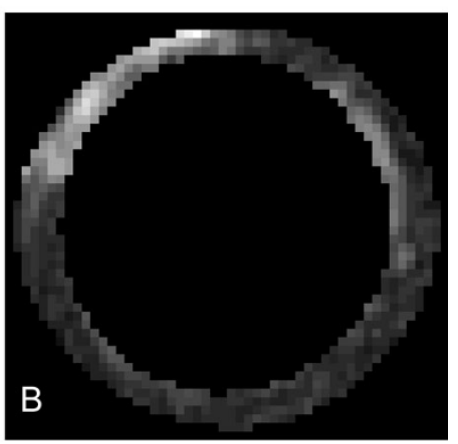

FWHM

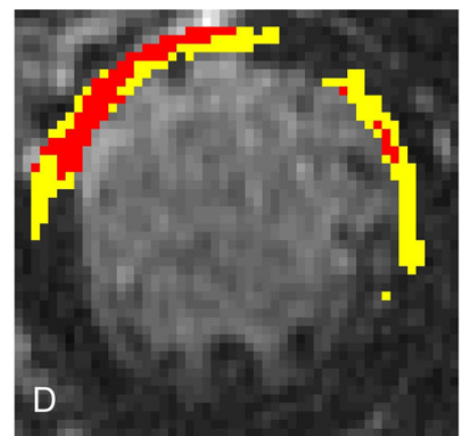

Figure 1 Infarct core and gray zone segmented with graph cuts compared with FWHM methods. A: Automated epicardial (red), endocardial (green) and remote myocardium(yellow). B: ROI Image. C: Infarct core (red) and gray zone(yellow) segmented with graph cuts (without remote myocardium). D: Infarct core (red) and gray zone (yellow) segmented with FWHM.
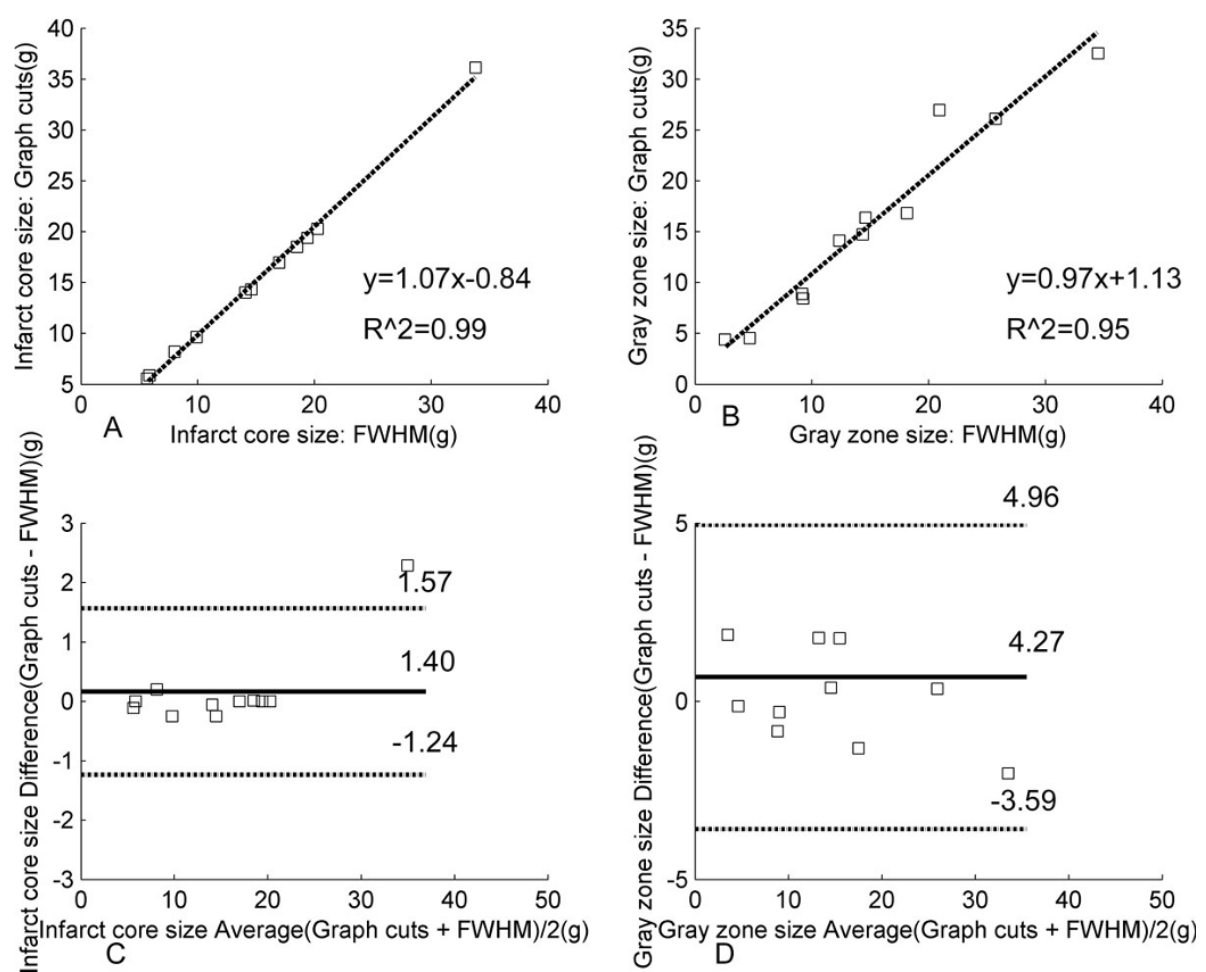

Figure 2 Linear regression between graph cuts and FWHM calculation of infarct mass for infarct core (A) and gray zone (B). Bland-Altman plots comparing graph cuts and FWHM calculations of infarct core (C) and gray zone (D). The horizontal solid line is bias, and dashed lines are the limits of agreement (+/- 1.96 SD). 


\section{Author details}

${ }^{1}$ Imaging Research, Sunnybrook Health Sciences Centre, Toronto, ON,

Canada. ${ }^{2}$ Keenan Research Centre in the Li Ka Shing Knowledge Institute, St. Michael's Hospital and University of Toronto, Toronto, ON, Canada.

${ }^{3}$ Cardiology, Sunnybrook Health Sciences Centre, Toronto, ON, Canada.

${ }^{4}$ Medical Biophysics, University of Toronto, Toronto, ON, Canada.

Published: 1 February 2012

\section{References}

1. Flett AS, et al: JACC: cardiovascular imaging. 2011, 4(2):150-156.

2. Roes SD, et al: Circulation: cardiovascular imaging. 2009, 2:183-190.

3. Lu Y, et al: Segmentation of Left Ventricle in Cardiac Cine MRI: An Automatic Image-Driven Method. Lecture Notes in Computer Science 2009, 5528/2009:339-347.

4. Boykov $Y$, et al: Fast approximate energy minimization via graph cuts. IEEE transactions PAMI 2001, 23(11):1222-1239.

doi:10.1186/1532-429X-14-S1-T6

Cite this article as: Lu et al:: Semi-automated analysis of infarct

heterogeneity on DE-MRI using graph cuts. Journal of Cardiovascular Magnetic Resonance 2012 14(Suppl 1):T6.

\section{Submit your next manuscript to BioMed Central} and take full advantage of:

- Convenient online submission

- Thorough peer review

- No space constraints or color figure charges

- Immediate publication on acceptance

- Inclusion in PubMed, CAS, Scopus and Google Scholar

- Research which is freely available for redistribution

Submit your manuscript at www.biomedcentral.com/submit
C Biomed Central 